\title{
Correction to: Non-rational action in the face of disagreement: an argument against (strong) non-conformism
}

\section{Nikolaj Jang Lee Linding Pedersen ${ }^{1,2}$}

Published online: 1 November 2018

(c) Springer Nature B.V. 2018

\section{Correction to: Synthese (2018) 195:2935-2966 https://doi.org/10.1007/s11229-016-1086-0}

Acknowledgements The Acknowledgements are missing from the original publication.

Nikolaj J. L. L. Pedersen gratefully acknowledges support from the National Research Foundation of Korea (Pluralisms Global Research Network, Grant No. 2013S1A2A203551).

The original article can be found online at https://doi.org/10.1007/s11229-016-1086-0.

$凶 \quad$ Nikolaj Jang Lee Linding Pedersen

nikolajpedersen@gmail.com; nikolaj@yonsei.ac.kr

1 Underwood International College, Yonsei University, Veritas B 427, Yonsei International Campus, 162-1 Songdo-dong, Yeonsu-gu, Incheon 406-840, South Korea

2 Veritas Research Center, Underwood International College, Yonsei University, Veritas B 427, Yonsei International Campus, 162-1 Songdo-dong, Yeonsu-gu, Incheon 406-840, South Korea 